\title{
Nonlinear dynamics in infant respiration
}

\author{
Michael SMaLl
}

By using inductance plethysmography it is possible to obtain a non-invasive measurement of the chest and abdominal cross-sectional area. These measurements are "representative" of the instantaneous lung volume. This thesis describes an analysis of the breathing patterns of human infants during quiet sleep using techniques of nonlinear dynamical systems theory $[\mathbf{1}, \mathbf{6}, \mathbf{7}]$. The purpose of this study is to determine if these techniques may be used to extend our understanding of the human respiratory system and its development during the first few months of life. Ultimately, we wish to use these techniques to detect and diagnose abnormalities and illness (such as apnea and sudden infant death syndrome) from recordings of respiratory effort during natural sleep.

Previous applications of dynamical systems theory to biological systems have been primarily concerned with the estimation of dynamic invariants: correlation dimension, Lyapunov exponents, entropy and algorithmic complexity. However, estimating these numbers has not proven useful in general. The study described in this thesis focuses on building models from time-series recordings and using these models to deduce properties of the underlying dynamical system. We apply a correlation dimension estimation algorithm $[2,3]$ in conjunction with well known surrogate data techniques $[16,17,18]$ and conclude that the respiratory system is not linear $[10,13]$. To elucidate the nature of the nonlinearity within this complex system we apply a new type of radial basis modelling algorithm (cylindrical basis modelling) $[4,5,9]$ and generate new nonlinear surrogate data $[10,11]$.

New nonlinear radial (cylindrical) basis modelling techniques have been developed by the author to model accurately this data [9]. This thesis presents new results concerning the use of correlation integral based statistics for surrogate data hypothesis testing. This extends the scope of surrogate data techniques to include hypotheses concerned with broad classes of nonlinear systems $[8, \mathbf{1 1}]$. We conclude that the human respiratory system behaves as a periodic oscillator with two or three degrees of freedom [13]. This

Received 21st June, 1999

Thesis submitted to the University of Western Australia, August 1998. Degree approved, February 1999. Supervisors Dr. K. Judd and Dr S. Stick.

Copyright Clearance Centre, Inc. Serial-fee code: 0004-9727/99 \$A2.00+0.00. 
system is shown to exhibit cyclic amplitude modulation (CAM) during quiet sleep [13, 15].

By examining the eigenvalues of fixed points exhibited by our models, and the qualitative features of the asymptotic behaviour of these models, we find further evidence to support this hypothesis [14]. An analysis of Poincare sections and the stability of the periodic orbits of these models demonstrates that CAM is present in models of almost all data sets. Models which do not exhibit CAM often exhibit chaotic first return maps. Some models are shown to exhibit period doubling bifurcations in the first return map.

To quantify the period and strength of CAM we suggest a new statistic based on an information theoretic reduction of linear models [12]. The models we utilise offer substantial simplification of autoregressive models and provide superior results. We show that the period of CAM present before a sigh and the period of subsequent periodic breathing are the same $[13,15]$. This suggests that CAM is ubiquitous but only evident during periodic breathing [13]. Physiologically, CAM may be linked to an autoresucitation mechanism. We observe a significantly increased incidence of CAM in infants at risk of sudden infant death syndrome and a higher incidence of CAM during apneaic episodes of bronchopulmonary dysplasic infants.

\section{REFERENCES}

[1] H.D.I. Abarbanel, Analysis of observed chaotic data, Institute for nonlinear science (Springer-Verlag, Berlin, Heidelberg, New York, 1996).

[2] K. Judd, 'An improved estimator of dimension and some comments on providing confidence intervals', Phys. D. 56 (1992), 216-228.

[3] K. Judd, 'Estimating dimension from small samples', Phys. D. 71 (1994), 421-429.

[4] K. Judd and A. Mees, 'On selecting models for nonlinear time series', Phys. D. 82 (1995), 426-444.

[5] K. Judd and A. Mees, 'Embedding as a modelling problem', Phys. D. 120 (1998), 273-286.

[6] H. Kantz and T. Schreiber, Nonlinear time series analysis, Cambridge Nonlinear Science Series 7 (Cambridge University Press, Cambridge, 1997).

[7] D. Kaplan and L. Glass, Understanding nonlinear dynamics, Texts in Applied Mathematics 19 (Springer-Verlag, Berlin, Heidelberg, New York, 1996).

[8] M. Small and K. Judd, 'Using surrogate data to test for nonlinearity in experimental data', in International Symposium on Nonlinear Theory and its Applications 2 (Research Society of Nonlinear Theory and its Applications, IEICE, 1997), pp. 1133-1136.

[9] M. Small and K. Judd, 'Comparison of new nonlinear modelling techniques with applications to infant respiration', Phys. D. 117 (1998), 283-298.

[10] M. Small and K. Judd, 'Detecting nonlinearity in experimental data', Internat. J. Bifur. Chaos Appl. Sci. Engrg. 8 (1998), 1231-1244.

[11] M. Small and K. Judd, 'Pivotal statistics for non-constrained realizations of composite null hypotheses in surrogate data analysis', Phys. D. 120 (1998), 386-400. 
[12] M. Small and K. Judd. 'Detecting periodicity in experimental data using linear modeling techniques', Phys. Rev. E. 59 (1999), 1379-1385.

[13] M. Small, K. Judd, M. Lowe and S. Stick, 'Is breathing in infants chaotic? Dimension estimates for respiratory patterns during quiet sleep', J. Appl. Physiol. 86 (1999), 359-376.

[14] M. Small, K. Judd and A. Mees, 'Testing time series for nonlinearity', Statistics and Computing (1999) (to appear).

[15] M. Small, K. Judd, and S. Stick, 'Linear modelling techniques detect periodic respiratory behaviour in infants during regular breathing in quiet sleep', (abstract), Am. J. Resp. Crit. Care. Med. 153 (1996), A79.

[16] F. Takens, 'Detecting nonlinearities in stationary time series', Internat. J. Bifur. Chaos Appl. Sci. Engrg. 3 (1993), 241-256.

[17] J. Theiler, S. Eubank, A. Longtin, B. Galdrikian and J.D. Farmer, 'Testing for nonlinearity in time series: The method of surrogate data', Phys. D. 58 (1992), 77-94.

[18] J. Theiler and D. Prichard, 'Constrained-realization Monte-Carlo method for hypothesis testing', Phys. D. 94 (1996), 221-235.

Centre for Applied Dynamics and Optimization Department of Mathematics and Statistics University of Western Australia

Nedlands WA 6907

Australia
Current Address:

Department of Physics

Heriot-Watt University

Riccarton, Edinburgh, EH14 4AS

United Kingdom

e-mail: M.A.Small@hw.ac.uk 